\title{
Left ventricular mass regression may occur very early following transcatheter aortic valve implantation for severe aortic stenosis
}

\author{
Laura E Dobson ${ }^{2}$, Tarique A Musa², Timothy A Fairbairn², Akhlaque Uddin², Daniel J Blackman', David P Ripley², \\ Peter P Swoboda ${ }^{2}$, Adam K McDiarmid², Bara Erhayiem², Pankaj Garg², Sven Plein², John P Greenwood ${ }^{2}$
}

From 18th Annual SCMR Scientific Sessions

Nice, France. 4-7 February 2015

\section{Background}

The effect of transcatheter aortic valve implantation (TAVR) on left ventricular remodelling early following valve implantation has not previously been evaluated using cardiac magnetic resonance (CMR). Early left ventricular mass (LVM) regression (assessed using echocardiography) has been linked to a $50 \%$ reduction in rehospitalisation in the first year following TAVR. We sought to establish using CMR, the reference standard non-invasive imaging technique for LVM quantification, whether LVM regression occurs early post-TAVR.

\section{Methods}

27 patients with symptomatic severe aortic stenosis undergoing TAVR were prospectively enrolled between June 2013 and April 2014. Patients with contraindications to CMR were excluded and all patients provided informed written consent. All patients underwent an identical 1.5T CMR protocol (Intera, Philips) prior to and at a median of 5 days following TAVR (IQR 2 days). Multislice, multi-phase cine imaging was carried out using a standard steady-state free procession pulse sequence in the short axis to cover the entire left ventricle. Quantitative analysis was performed using dedicated computer software $\left(\mathrm{CVI}^{42}\right.$, Circle Cardiovascular Imaging, Alberta, Canada). LVM was calculated by the formula (epicardial volume - endocardial volume) $\mathrm{x}$ myocardial density $\left(1.05 \mathrm{~g} / \mathrm{cm}^{3}\right)$.

\section{Results}

Basic patient, echocardiographic and procedural characteristics can be seen in Table 1 .

At a median of 5 days following TAVR, mean LVM regressed by approximately $12 \%$ from $129.5 \pm 32.5 \mathrm{~g}$ to $114.2 \pm 31 \mathrm{~g}$ and indexed LVM (LVMi) reduced from 69.4 $\pm 15.2 \mathrm{~g} / \mathrm{m}^{2}$ to $61.3 \pm 15.1 \mathrm{~g} / \mathrm{m}^{2}$ (Graph 1 ). There was no significant change in indexed left ventricular diastolic volume $(96.9 \pm 24.5$ to $96.8 \pm 19.3 \mathrm{ml} / \mathrm{m} 2, \mathrm{p}=0.79)$, $\mathrm{LV}$ ejection fraction (54.0 \pm 14.6 to $58.4 \pm 27.4 \%, \mathrm{p}=0.49)$ or indexed left atrial volume $(71.5 \pm 22.6$ to $67.7 \pm 22.1 \mathrm{ml} /$ $\mathrm{m} 2, \mathrm{p}=0.43)$. LVM regression was similar between the Medtronic and Boston valve types $(14.3 \pm 10.4 \mathrm{~g}$ Vs $16.5 \pm$ $9.9 \mathrm{~g}, \mathrm{p}=0.58)$. There was no correlation between Valvulo-arterial impedance (ZVA) and LVM regression $(\mathrm{r}=0.02, \mathrm{p}=0.93)$. There was no difference in mean LVMi regression according to gender (women $8.3 \pm 2.8 \mathrm{~g} /$ $\mathrm{m}^{2}$ Vs men $8.0 \pm 5.7 \mathrm{~g} / \mathrm{m}^{2}, \mathrm{p}=0.83$ ). TAVR valve size did not appear to impact on early LVM regression. LVMi regression was similar between those with a normal baseline LVM and those with an elevated baseline LVM (defined as $83 \mathrm{~g} / \mathrm{m}^{2}$ in men and $67 \mathrm{~g} / \mathrm{m}^{2}$ ) in women $(7.8$ $\pm 4.8 \mathrm{~g}$ Vs $9.0 \pm 5.7 \mathrm{~g}, \mathrm{p}=0.60$ ).

16 patients had evidence of late gadolinium enhancement (LGE) at baseline. There was a trend towards increased LVMi regression in those without LGE at baseline compared with those with LGE $\left(10.1 \pm 4.7 \mathrm{~g} / \mathrm{m}^{2}\right.$ Vs $\left.6.4 \pm 4.7 \mathrm{~g} / \mathrm{m}^{2}, \mathrm{p}=0.07\right)$.

\section{Conclusions}

LVM regression begins very early following TAVR and occurs before changes in cavity size or ejection fraction which is likely to be a result of an immediate reduction 
Table 1 Patient, echocardiographic and procedural characteristics. Mean \pm SD.

\begin{tabular}{cc}
\hline Age, $y$ & $78 \pm 6$ \\
\hline Gender, (male \%) & $19(70 \%)$ \\
\hline STS Mortality score, \% & $4.1 \pm 3$ \\
\hline STS Morbidity/mortality score, \% & $21.6 \pm 7$ \\
\hline Hypertension (\%) & $12(44)$ \\
\hline NYHA Grade & $2.9 \pm 0.5$ \\
\hline AVA (cm2) & $0.62 \pm 0.21$ \\
\hline Peak pressure drop (mmHg) & $90 \pm 27$ \\
\hline TAVI Type & $14(52)$ \\
\hline Medtronic Corevalve (\%) & $12(44)$ \\
\hline Boston Lotus (\%) & $1(4)$
\end{tabular}

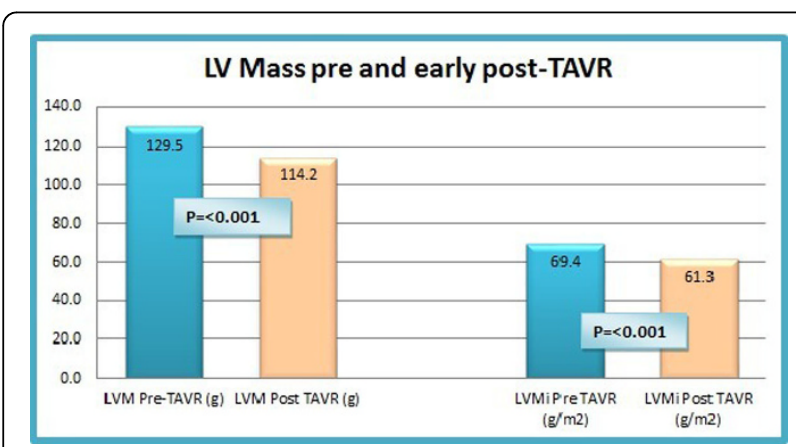

Figure 1

in wall stress. The type of valve implanted does not appear to influence early LVM regression.

\section{Funding}

This study was part funded by the British Heart Foundation (PG/11/126/29321).

\section{Authors' details}

${ }^{1}$ Yorkshire Heart Centre, Leeds Teaching Hospitals Trust, Leeds, UK. ${ }^{2}$ Cardiac MRI, Multidisciplinary Cardiovascular Research Centre \& Leeds institute of Cardiovascular and Metabolic Medicine, Leeds, UK.

Published: 3 February 2015

doi:10.1186/1532-429X-17-S1-P341

Cite this article as: Dobson et al.: Left ventricular mass regression may occur very early following transcatheter aortic valve implantation for severe aortic stenosis. Journal of Cardiovascular Magnetic Resonance 2015 17(Suppl 1):P341.

Submit your next manuscript to BioMed Central and take full advantage of:

- Convenient online submission

- Thorough peer review

- No space constraints or color figure charges

- Immediate publication on acceptance

- Inclusion in PubMed, CAS, Scopus and Google Scholar

- Research which is freely available for redistribution

Submit your manuscript at www.biomedcentral.com/submit 